\section{Ovarian Cancer- Is there Hope for Women through Screening?}

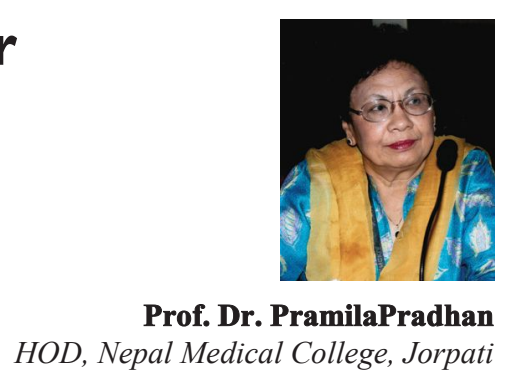

Ovarian Cancer today is the most lethal cancer of the female pelvis with an overall survival of only $30 \%$ largely due to the advanced stage at presentation of the disease. Early Stage disease is generally difficult to diagnose due to non-specific symptoms. Whether population-based screening can effectively diagnose early-stage disease and thereby impact upon long term outcome remains one of the current challenges in Gynaecological Oncology.

The WHO recommends that population-based screening programs are worthwhile only for conditions that have high population prevalence, are major causes of mortality and have a well defined natural history where intervention in early-stage disease is likely to have positive impact on outcome. They recommend that screening tests should be acceptable, easy to implement, cost-effective and that they should have a high sensitivity, specificity, positive and negative predictive value (PPV and NPV).

Conventionally population-based screening for ovarian cancer has relied on using CA 125 level, transvaginal pelvic ultrasound (with or without colour Doppler Study of Ovarian Blood Flow) either alone or in combination. The ideal screening test for ovarian cancer should have sensitivity greater than $75 \%$, specificity of $99 \%$ and a positive predictive value more than $10 \%$ i.e. 10 surgeries performed per detected cancer ${ }^{1}$.Serum CA 125 is however very non-specific as a marker for ovarian cancer and levels are found to be elevated in a number of gynaecological and non-gynaecological conditions, when used a single test CA 125 has a sensitivity of approximately $80 \%$ and specificity of $75 \%$. Van Nagell et al used TVS as a screening tool in asymptomatic post menopausal women and showed that although TVS has acceptable sensitivity, the positive predictive value (PPV) was very low. The reported sensitivity of $81 \%$, specificity of $98.4 \%$ and PPV of $9.4 \%$ is not regarded as acceptable per population-based screening. Attempts have been made to improve the performance of TVS as a screening test by using other ultrasound based tests e.g. Morphology indexing and Doppler flow studies which has a sensitivity of $98 \%$, a specificity of $80.1 \%$, PPV of $40.9 \%$ and NPV of $99.7 \%$.Colour Doppler Studies of Ovarian Blood Flow does not appear to offer any additional advantage in improving the performance of TVS as a screening test.

In recent years there has been some interest in evaluating novel tumour markers in addition to CA 125 and these include Lysophosphatidicacid (LPA), CA 72-4, Macrophage-Colony Stimulating Factor (M-CSF), Osteopontin, Inhibin and Kallikrein ${ }^{2}$. Published data to-date are sparse and not encouraging.

Three large randomised controlled multi-modality screening trials have been published till today. Japanese trial by H. Kobayashi et al, The United Kingdom Collaborative Ovarian Cancer Screening (UKCTOCS) and prostate, lung, colorectal and ovarian cancer (PLCO) trial. All the randomised trial suggest that there is no robust evidence to recommend routine population-based screening for sporadic ovarian cancer in low risk asymptomatic women with no family history of ovarian or breast cancer. The rationale being that the PPV with CA 125 or TVS is less that $3 \%$ and false positive rates are high. Surgical intervention rates are high and there is no evidence

Correspondence

Prof. Dr. PramilaPradhan MRCOG

HOD OBS / GYN

Nepal Medical College, Jorpati

Mobile :9841490496

E-mail:drpramilapradhan@gmail.com 
that such screening actually reduce mortality from ovarian cancer.

The life time risk of developing ovarian cancer for women in the general population is 1.8 increasing to $30-50 \%$ in women with familial ovarian cancer syndrome ${ }^{3}$. So, the question here is who do we screen? It is recommended that high risk women who have a history of 2 or more ovarian cancer in a first degree relative, one ovarian and one breast cancer $(<50 \mathrm{yrs})$ in a first degree relative, one ovarian and two breast cancer $(<60 \mathrm{yrs})$ in a first degree relative, one ovarian and 3 colorectal cancers (at least one $<50 \mathrm{yrs}$ ) in a first degree relative or if there is a documented mutation in a predisposing gene i.e. BRCA 1 or BRCA 2 should be screened 6 monthly with pelvic examination, TVS +/- Doppler Study, Serum CA 125. Prophylactic BSO after completion of child bearing or at age 35 may be $\operatorname{advised}^{1,3}$.

Whether screening is really effective even in high risk women is a question that is still open to debate. The United Kingdom Family Ovarian Cancer Screening Study (UKFOCSS) aims to detect the efficacy of screening vis-a-visit's ability to detect early stage disease. This study includes only high risk women for ovarian cancer. Preliminary data suggest excellent compliance for this study and results of UKFOCSS are awaited.

The current available screening modalities are disappointing in detecting highly curable early stage ovarian cancer in an asymptomatic woman. Unlike cervical cancer, the natural history of ovarian cancer is still not clear. Reports of normal looking ovaries on ultrasounds or laparoscopy just a few months prior to diagnosis of advanced ovarian cancer suggests that ovarian cancer can develop relatively quickly from normal looking ovaries. As most women with ovarian cancer complain of non-specific symptoms many months prior to their diagnosis significant delay could be avoided by timely referral for multimodality screening test.

\section{References}

1. Clark-Pearson DL. Screening for Ovarian Cancer. N Eng J Med 2009;361: 1701-7

2. Visintin I, Feng Z, Longton G et al. Diagnostic markers for early detection of ovarian cancer. Clin Can Res 2008; 14: 1065-72 [ Errata Clin Cancer Res 2008; 14: 5308, 7158]

3. National Comprehensive Cancer Network $(\mathrm{NCCN})$ Practice Guidelines in Oncology. 